Check for updates

Cite this: RSC Adv., 2019, 9, 20424

\title{
Facile synthesis of Camellia oleifera shell-derived hard carbon as an anode material for lithium-ion batteries $\dagger$
}

\author{
Beibei Ma, ${ }^{a}$ Yewei Huang, ${ }^{a}$ Zhenzhen Nie, ${ }^{a}$ Xiaobin Qiu, ${ }^{a}$ Dawei Su, (D) *b \\ Guoxiu Wang, (D)*b Jianmin Yuan, ${ }^{c}$ Xiuqiang Xie (D) ${ }^{c}$ and Zhenjun Wu (D) *a
}

\begin{abstract}
A comparatively facile and ecofriendly process has been developed to synthesize porous carbon materials from Camellia oleifera shells. Potassium carbonate solution $\left(\mathrm{K}_{2} \mathrm{CO}_{3}\right)$ impregnation is introduced to modify the functional groups on the surface of Camellia oleifera shells, which may play a role in promoting the development of pore structure during carbonization treatment. Moreover, a small amount of naturally embedded nitrogen and sulfur in the Camellia oleifera shells can also bring about the formation of pores. The Camellia oleifera shell-derived carbon has a large specific surface area of $1479 \mathrm{~m}^{2} \mathrm{~g}^{-1}$ with a total pore volume of $0.832 \mathrm{~cm}^{3} \mathrm{~g}^{-1}$ after being carbonized at $900{ }^{\circ} \mathrm{C}$ for $1 \mathrm{~h}$. Furthermore, when used as an anode for lithium-ion batteries, the sample shows superior electrochemical performance with a specific capacity of $483 \mathrm{~mA} \mathrm{~h} \mathrm{~g}{ }^{-1}$ after 100 cycles measured at $200 \mathrm{~mA} \mathrm{~g}^{-1}$ current density. Surprisingly, the specific capacity is even gradually increased with cycling. In addition, this sample exhibits almost $100 \%$ retention capacity after 250 cycles at a current density of $200 \mathrm{~mA} \mathrm{~g}^{-1}$.
\end{abstract}

Received 5th May 2019

Accepted 25th June 2019

DOI: 10.1039/c9ra03345a

rsc.li/rsc-advances rising. On the other hand, graphite is the most widely used anode for commercial rechargeable LIBs. However, confined by its limited storage capacity $\left(372 \mathrm{~mA} \mathrm{~h} \mathrm{~g}^{-1}\right)$ and low rate performance, graphite cannot satisfy the urgent demand for high-performance LIBs. $^{7,8}$ In consideration of the cost of electrochemical energy storage systems, sodium ion batteries (NIBs), which use sodium as the counter electrode, have attracted great attention. Sodium is cheap, abundant and widely available with respect to its lithium counterpart. However, finding suitable anode materials for sodium ion batteries has plagued many scientists. The ionic radius of $\mathrm{Na}^{+}(102 \mathrm{~nm})$ is larger than that of $\mathrm{Li}^{+}(76 \mathrm{~nm})$, which leads to poor kinetics in the $\mathrm{Na}^{+}$(de)insertion reaction, further deteriorating the cycling performance and rate performance of NIBs. ${ }^{9,10}$ Therefore, in such a scenario, the exploitation of advanced active anode materials with high performance is of particular importance. In addition, ideal electrode materials should be obtained from renewable resources in an efficient manner, taking into account environmental issues and economic value. .112 $^{1,12}$

In the context of the materials used, biomass-derived hard carbon materials, which are abundant, easily accessible, low cost and renewable, ${ }^{\mathbf{1 3}}$ have attracted extensive attention in the fabrication of high-performance anodes for LIBs. Moreover, due to the porosity of these biomass carbon materials, they can provide a wide channel for the charge-transfer reaction and facilitate ion transport by shortening diffusion pathways. ${ }^{\mathbf{1 4}}$ Accordingly, in recent years, porous carbons derived from biomass for LIB anodes have been explored with a wide range of sources, such as olive, ${ }^{15,16}$ cherry stone, ${ }^{7,15}$ water hyacinth,,${ }^{17}$ rice husk, ${ }^{18}$ coconut oil, ${ }^{19}$ peanut shell, ${ }^{20}$ sweet potato, ${ }^{21}$ cotton, ${ }^{22}$ 
litchi, ${ }^{23}$ ramie fiber and corncob. ${ }^{24}$ In one study, byproduct rice husk was used as a carbon precursor and applied as an anode for LIBs, presenting a capacity of $403 \mathrm{~mA} \mathrm{~h} \mathrm{~g}^{-1}$ after 100 cycles at a current density of $75 \mathrm{~mA} \mathrm{~g}^{-1}$, higher than that of commercial graphite anodes. ${ }^{18}$ Similarly, a N-self-doped hierarchically porous carbon from the stem medulla of water hyacinth was synthesized by a facile self-activation approach, exhibiting excellent cycle performance and rate capacity when used as an anode in LIBs. ${ }^{17}$

Camellia oleifera shells are byproducts of camellia oil and are produced in large amounts in Hunan Province, which is one of the largest cultivation areas of Camellia oleifera in China. Moreover, the Camellia oleifera industry has been established as a "First livelihood project" in Hunan Province to overcome poverty. This means that more Camellia oleifera shells after pressing oil will be produced as waste materials. However, the shells, notably, are not only rich in hemicellulose and cellulose, which are excellent sources of carbon, but also comprise a bit of naturally embedded nitrogen and sulfur. The chemical composition of the raw material is listed in Table 1. To date, studies on the preparation of porous carbon from Camellia oleifera shells have rarely been reported. It has been identified that biomassderived porous carbons can be activated with activating agents such as $\mathrm{KOH},{ }^{25-28} \mathrm{NaOH}^{29-31}$ and $\mathrm{ZnCl}_{2} \cdot{ }^{32-34}$ However, the strong basicity and toxic features of such agents make them hazardous for application in practice. Here, we propose a novel synthesis approach for porous carbon from Camellia oleifera shells (COSDHCs) activated by using $\mathrm{K}_{2} \mathrm{CO}_{3}$ as an activation agent, which is inexpensive and nontoxic. ${ }^{35}$ When the as-prepared COSDHCs were used as an anode material for LIBs, they achieved a high specific capacity of $483 \mathrm{~mA} \mathrm{~h} \mathrm{~g}^{-1}$ after 100 cycles. Surprisingly, almost $100 \%$ retention capacity after 250 cycles at a current density of $200 \mathrm{~mA} \mathrm{~g}^{-1}$ was measured. The excellent electrochemical performance of the as-prepared COSDHCs can be ascribed to their large specific surface area of $1479 \mathrm{~m}^{2} \mathrm{~g}^{-1}$ and high pore volume of $0.832 \mathrm{~cm}^{3} \mathrm{~g}^{-1}$ after activation by $\mathrm{K}_{2} \mathrm{CO}_{3}$, which benefits the volume expansion of the COSDHCs in the lithiation and delithiation processes during charge and discharge cycling. Furthermore, the porous architecture can promote electrolyte diffusion within a local environment, which can enhance the Li-ion kinetics for reaction with COSDHCs, as verified by $\mathrm{CV}$ and impedance measurements.

\section{Experimental section}

\subsection{Material synthesis}

Camellia oleifera shells obtained from an oil mill in Hunan were first washed with ethanol and then dried at $80{ }^{\circ} \mathrm{C}$ overnight, followed by grinding into a fine powder by mechanical milling.

Table 1 Chemical compositions of the raw material and carbon

Elemental analysis (wt\%)

\begin{tabular}{lllll} 
& \multicolumn{4}{l}{ Elemental analysis (wt\%) } \\
\cline { 2 - 5 } Sample & $\mathrm{C}$ & $\mathrm{N}$ & $\mathrm{S}$ & $\mathrm{H}$ \\
\hline Raw material & 44.32 & 0.35 & 0.19 & 5.62 \\
COSDHC900-1 & 72.59 & 0.45 & 0.17 & 2.26
\end{tabular}

Then, $2 \mathrm{~g}$ potassium carbonate $\left(\mathrm{K}_{2} \mathrm{CO}_{3}\right)$ was dissolved in $8 \mathrm{~mL}$ deionized water to prepare a clear solution. A total of $1.5 \mathrm{~g}$ of the Camellia oleifera shell powder was immersed into the solution, stirred overnight and dried at $120{ }^{\circ} \mathrm{C}$ for $6 \mathrm{~h}$ in an air drying oven. Finally, the obtained samples were milled and prepared by pyrolysis in a tubular furnace at a heating rate of $5{ }^{\circ} \mathrm{C} \mathrm{min}^{-1}$ under Ar atmosphere. The samples are denoted as COSDHC- $T$ $H$, where $T$ and $H$ represent the pyrolysis temperature and carbonization time, respectively; samples were carbonized at 800 and $900{ }^{\circ} \mathrm{C}$ for 1 and/or $2 \mathrm{~h}$ to yield COSDHC800-1, COSDHC800-2 and COSDHC900-1. After carbonization, the samples were boiled for $20 \mathrm{~min}$ in $20 \mathrm{wt} \% \mathrm{HCl}$ to remove any residual impurities, sequentially washed with hot water several times until the solution was neutral and dried at $80^{\circ} \mathrm{C}$ overnight in a vacuum oven.

\subsection{Material characterization}

The raw material and as-prepared samples were subjected to various forms of characterization. The distribution of the major elements in the raw material and carbonized product was determined by elemental analysis (VARIO ELIII, Germany) and X-ray photoelectron spectroscopy (XPS, SCALAB 250Xi, Thermo Fisher Scientific, America). Thermogravimetric analysis of the impregnated sample was carried out with a Netzsch STA 409PC thermal analyzer at a heating rate of $5{ }^{\circ} \mathrm{C} \mathrm{min}^{-1}$ from room temperature to $1000{ }^{\circ} \mathrm{C}$ under $\mathrm{N}_{2}$ atmosphere. The morphology of the synthesized carbon materials was observed using scanning electron microscopy (SEM, Hitachi, S-4800), transmission electron microscopy (TEM, FEI Tecnai G20) operating at $200 \mathrm{kV}$ and high-resolution transmission electron microscopy (HRTEM). X-ray diffraction patterns (XRD, Bruker D8 Advance), Raman spectroscopy (Renishaw Invia-reflex, $532 \mathrm{~nm}$ laser) and Fourier transform infrared spectroscopy (FT-IR, IR Affinity) were used to examine the degree of graphitization, crystallinity, and functional groups. The surface area was characterized by nitrogen adsorption at $200{ }^{\circ} \mathrm{C}$ with a Quantachrome NOVA1000e apparatus, and the pore size distributions were analyzed via the Barrett-Joyner-Halenda (BJH) method.

\subsection{Electrochemical measurements}

The electrode slurry was formulated from a mixture of $80 \mathrm{wt} \%$ as-prepared powder, $10 \mathrm{wt} \%$ super carbon black, and $10 \mathrm{wt} \%$ polymer binder (polyvinylidene fluoride (PVDF)) using $N$-methyl pyrrolidinone (NMP) as the dissolving solvent. Then, the carbon-coated COSDHC electrodes were mechanically pressed and assembled into CR2032 coin cells with lithium foil as the counter and reference electrodes. The LIB electrolyte and separator were $1 \mathrm{M} \mathrm{LiPF}_{6}$ in a mixture of ethylene carbonate and diethyl carbonate at a volume ratio of $1: 1$ and a polyethene separator (Celgard 2400), respectively. All test cells were assembled in an Ar-filled glovebox with sub-0.1 ppm water and oxygen contents. The galvanostatic charge/discharge measurements were performed on a Land CT2001A system (Wuhan, China) with a cutoff voltage window of $0.01-3 \mathrm{~V}\left(v s . \mathrm{Li} / \mathrm{Li}^{+}\right)$at $25{ }^{\circ} \mathrm{C}$. The cyclic voltammetry $(\mathrm{CV})$ curves were obtained on an Autolab PGSTAT302N (Metrohm-Autolab BV, Utrecht, The 
Netherlands) electrochemical workstation with a sweep rate of $0.1 \mathrm{mV} \mathrm{s}^{-1}$. Electrochemical impedance spectroscopy (EIS) was measured in the frequency range from $100 \mathrm{kHz}$ to $0.01 \mathrm{~Hz}$ at $5 \mathrm{mV} \mathrm{s}^{-1}$.

\section{Results and discussion}

The COSDHCs were prepared by impregnation and carbonization, and the synthetic route of this method is shown in Fig. 1. The pyrolysis process of the precursor (the impregnated Camellia oleifera shell powder) was examined by thermal analyzer. The TG curve is shown in Fig. S1. $\dagger$ A significant weight loss of $29 \mathrm{wt} \%$ was caused by the evaporation of residual water and the decomposition of hemicellulose, cellulose and even lignin stored in the Camellia oleifera shell. ${ }^{36}$ Another 55.2 wt\% mass loss may be due to decomposition and reduction reactions of $\mathrm{K}_{2} \mathrm{CO}_{3} \cdot{ }^{37}$ Then, infrared spectroscopy was used to determine the changes in functional groups. Fig. 2 displays the spectra of the raw material, the sample impregnated with $\mathrm{K}_{2} \mathrm{CO}_{3}$ and its char annealed at $900{ }^{\circ} \mathrm{C}$. The impregnated Camellia oleifera shell powder (IMPCOS) was carbonized under an Ar atmosphere at a rate of $5{ }^{\circ} \mathrm{C} \mathrm{min}{ }^{-1}$ from room temperature to $900{ }^{\circ} \mathrm{C}$ and held for $1 \mathrm{~h}$ (COSDHC900-1). ${ }^{38}$ When comparing the three spectra, it can be seen that, in addition to the carbon spectrum, the spectra show a strong broad absorption band at $\sim 3420 \mathrm{~cm}^{-1}$, which is assigned to the stretching vibration of phenolic hydroxyl or alcoholic hydroxyl groups. ${ }^{39}$ The absorption peaks at 2900 and $2880 \mathrm{~cm}^{-1}$ can be attributed to the $\mathrm{C}-\mathrm{H}$ stretching vibration of lignin polysaccharides. The $\mathrm{C}-\mathrm{H}$ stretching vibration is weakened in the high-temperature (900 ${ }^{\circ} \mathrm{C}$ ) carbonized materials, which may be due to the decomposition of lignin polysaccharides in the Camellia oleifera shells, such as cellulose and hemicelluloses. ${ }^{40}$ The peaks at $2360 \mathrm{~cm}^{-1}$ and $1750 \mathrm{~cm}^{-1}$ are ascribed to $\mathrm{O}=\mathrm{C}=\mathrm{O}$ vibrations and the $\mathrm{C}=\mathrm{O}$ bond stretching vibration of oxygen-containing functional groups, such as carboxyl groups, carbonyl groups and ester groups. ${ }^{38,41}$ After infiltrating $\mathrm{K}_{2} \mathrm{CO}_{3}$, the peaks corresponding to oxygen-containing functional groups are weakened, because of the effect of $\mathrm{K}_{2} \mathrm{CO}_{3}$ during the impregnation process. ${ }^{35}$ The $\mathrm{C}=\mathrm{O}$ band almost disappeared in the infrared spectrum of the

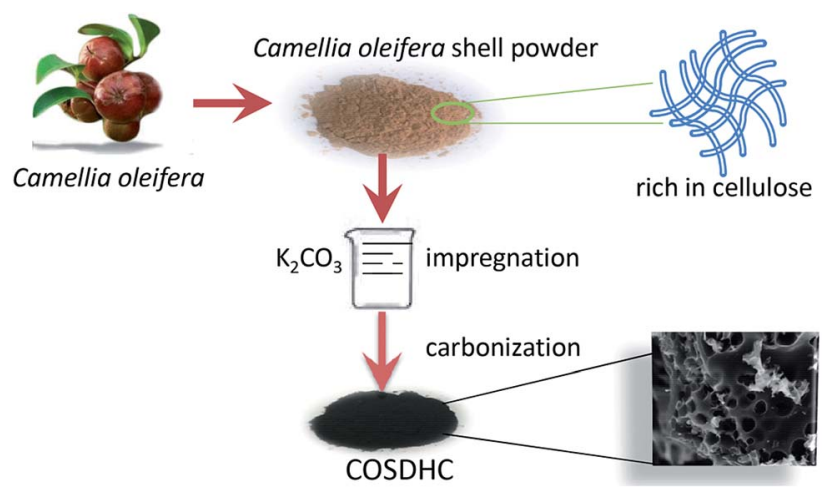

Fig. 1 The synthesis of porous hard carbons from Camellia oleifera shell.

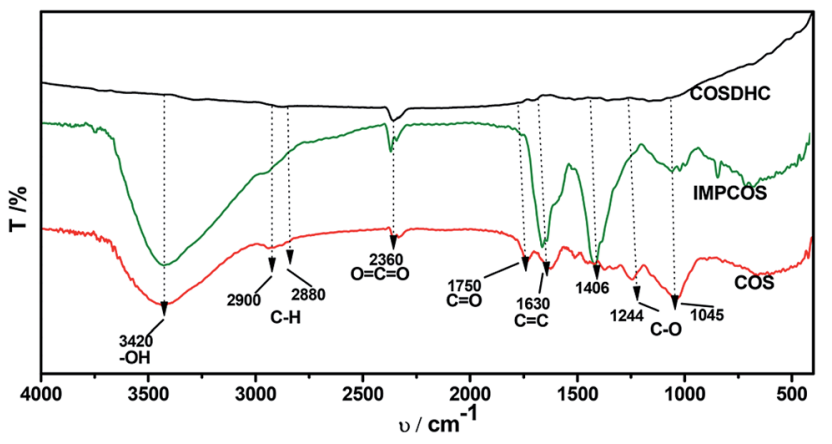

Fig. 2 FT-IR spectra of the raw material, impregnated sample and carbonized product.

biomass carbon produced at $900{ }^{\circ} \mathrm{C}$, confirming that $\mathrm{C}=\mathrm{O}$ bonds are more likely to be broken, resulting in the formation of $\mathrm{CO}$ and $\mathrm{CO}_{2}$. A strong band is observed at approximately $1630 \mathrm{~cm}^{-1}$, which may be generated by the aromatic skeleton region of the Camellia oleifera shell powder or $\mathrm{C}=\mathrm{C}$ stretching vibration, indicating that the biomass carbon gradually forms an aromatic structure. ${ }^{42}$ When the temperature is higher than $700{ }^{\circ} \mathrm{C}$, carbon in the coke is removed as $\mathrm{CO}$ gas by $\mathrm{K}_{2} \mathrm{CO}_{3}$ reduction. ${ }^{35,37}$ The band is greatly attenuated for the carbonized product. The peaks located at $1400-1000 \mathrm{~cm}^{-1}$ are attributed to $\mathrm{C}-\mathrm{O}$ stretching in alcohols, phenols, ethers and esters. These broad bands are unique to the COS and impregnated samples; nevertheless, the bands almost vanish for COSDHC900-1. This clearly indicates that the $\mathrm{C}-\mathrm{O}$ bonds in acids, alcohols, phenols, ethers and esters disappear during the carbonization process. ${ }^{43}$ Overall, the $\mathrm{C}=\mathrm{C}$ and $\mathrm{C}-\mathrm{O}$ intensities of Camellia oleifera shells dipped in $\mathrm{K}_{2} \mathrm{CO}_{3}$ solution are significantly higher than those of the corresponding raw materials and activated carbon samples. This contributes to the degree of aromatization of biomass carbon and tendency to form graphite crystallites. In addition, the amounts of functional groups are greatly reduced, which is also conducive to porosity.

The impregnated samples carbonized at $800^{\circ} \mathrm{C}$ for 1 and $2 \mathrm{~h}$ and $900{ }^{\circ} \mathrm{C}$ for $1 \mathrm{~h}$ were characterized and compared. As shown in the scanning electron microscopy (SEM) images, a large amount of cracked macropores and mesopores were generated in the samples carbonized at $800{ }^{\circ} \mathrm{C}$ (Fig. S2 $\dagger$ ). In comparison, the pores of COSDHC900-1 are more evenly distributed, and the surface presents a well-developed cross-linked mesoporous structure with thin walls. These pores can remarkably enhance the active sites for Li-ion storage. Moreover, the interconnected structure formed by the thin walls can act as an ion-buffering reservoir. Furthermore, such a cross-linked mesoporous structure immersed in electrolyte will provide short Li-ion transport and accelerate electrolyte diffusion (Fig. 3a and b). ${ }^{\mathbf{4 4}}$ These highly developed pores are due to the release of CO during the reduction of $\mathrm{K}_{2} \mathrm{CO}_{3}$ in the pyrolysis process. ${ }^{35,41}$ The typical transmission electron microscopy (TEM) image is shown in Fig. 3c. It is obvious that hierarchical micro- and mesopores are distributed in COSDHC900-1, which can serve as Li-ion containers to increase ion storage. ${ }^{45}$ In the high-resolution TEM image (Fig. 3d), COSDHC900-1 has an amorphous 


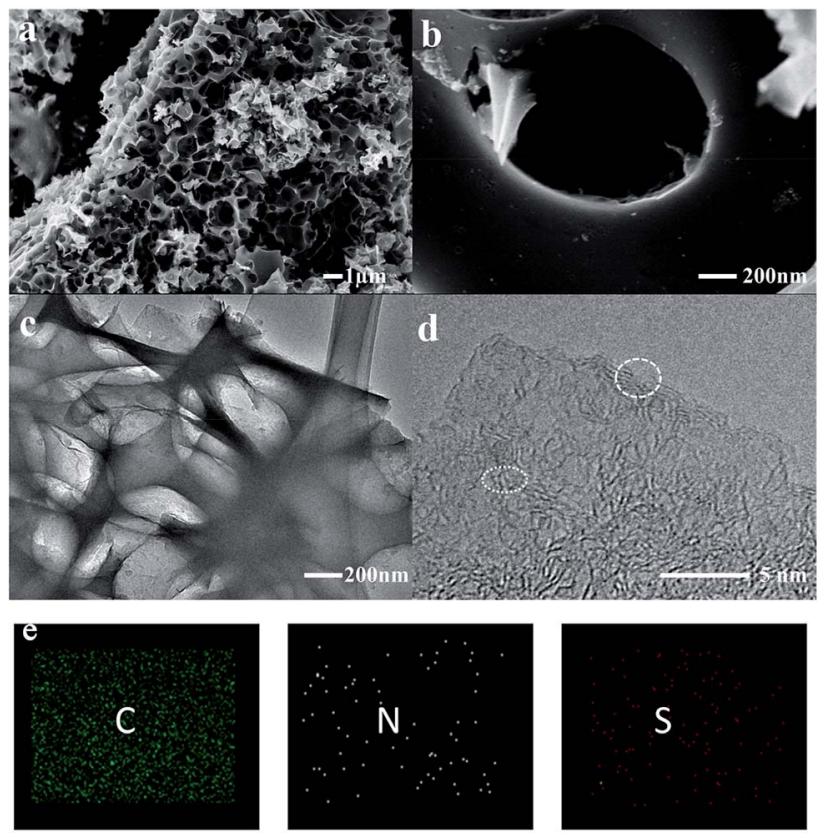

Fig. 3 Characterization of the COSDHC900-1: (a and b) SEM images, (c) TEM image, (d) high-resolution TEM image, (e) element mapping of C, N, and S

structure with some small domains of graphite microcrystallites in a short range. The elemental mapping images in Fig. 3e show that Camellia oleifera shells are indeed carbon-rich precursors and contain a small amount of nitrogen and sulfur. X-ray photoelectron spectroscopy (XPS) of the raw material and the sample calcined at $900{ }^{\circ} \mathrm{C}$ was further conducted to detect the surface element features (see Fig. S3 $\dagger$ ). Three peaks, centered at approximately $168 \mathrm{eV}, 285 \mathrm{eV}$ and $400 \mathrm{eV}$, correspond to S 2p, C 1s and $\mathrm{N} 1 \mathrm{~s}$, respectively. ${ }^{46}$ The $\mathrm{N}$ and $\mathrm{S}$ contents of COS and COSDHC are quite low with a sparse distribution. Very small amounts of nitrogen and sulfur atoms can also create defects in carbon materials because of their larger radius than $\mathrm{C}$ atoms and generate active sites for Li storage. ${ }^{47,48}$ These results are consistent with those of the element analysis (Table 1).

To further investigate the porosity in detail, the three samples were measured by nitrogen adsorption-desorption isotherms (Fig. 4a), and the corresponding pore size distribution curves are shown in Fig. 4b. The isotherms are revealed to be type I, according to IUPAC classification. There exist slight
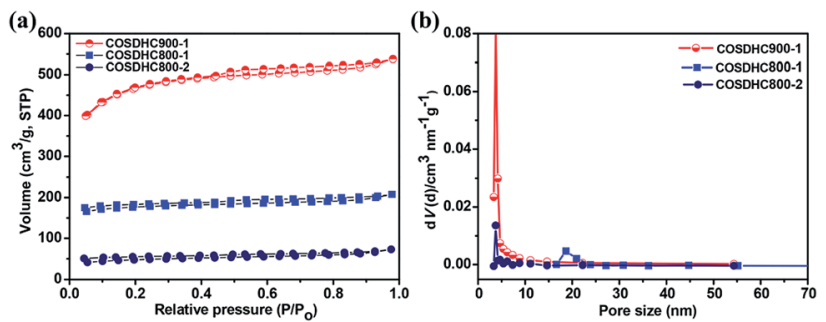

Fig. 4 (a) Nitrogen-sorption isotherms and (b) pore size distributions obtained for COSDHC900-1, COSDHC800-1 and COSDHC800-2.
H4 hysteresis loops. ${ }^{49}$ These loops indicate a series of hierarchical pores, including micropores, mesopores, and a small amount of large pores, in the samples. The surface areas of COSDHC900-1, COSDHC800-1, and COSDHC800-2, calculated by the Brunauer-Emmett-Teller (BET) method, are approximately $1479 \mathrm{~m}^{2} \mathrm{~g}^{-1}, 545 \mathrm{~m}^{2} \mathrm{~g}^{-1}$ and $152 \mathrm{~m}^{2} \mathrm{~g}^{-1}$, respectively. The detailed results of the Barrett-Joyner-Halenda (BJH) pore size distribution are summarized in Table 2. Among the three as-obtained samples, COSDHC900-1 has the highest specific area and pore volume, benefiting from the combination of micro- and mesopores at the relatively high pyrolysis temperature. Micropores contribute greatly to the surface exposure, while the mesopores mainly contribute to the total pore volume. For COSDHC900-1, the pore volume and average pore diameter are $0.832 \mathrm{~cm}^{3} \mathrm{~g}^{-1}$ and $2.25 \mathrm{~nm}$, respectively. These slit-shaped (or cylindrical) and narrow-mouthed pores were primarily produced by $\mathrm{CO}$ and $\mathrm{K}$ release when the activation temperature reached the melting point of $\mathrm{K}_{2} \mathrm{CO}_{3}$ at $891{ }^{\circ} \mathrm{C} .{ }^{37}$ During the carbonization and activation process, $\mathrm{K}_{2} \mathrm{CO}_{3}$ is reduced by the non-graphitic carbon $^{50}$ under inert gas flow, resulting in micropores, and then the existing micropores are enlarged in the carbonization process at that temperature. This leads to the combination of mesopores and micropores within the carbon framework. This unique porous structure with remarkable surface area and large pore volume can provide more adsorption sites for Li ions and electrolyte infiltration to achieve fast ion transport and short diffusion.

The crystal structure of the samples at different temperatures and carbonization periods was characterized by X-ray diffraction (XRD). As shown in Fig. 5a, two main broad diffraction peaks at $20^{\circ}$ and $44^{\circ}$ appear in all samples, corresponding to the (002) and (100) lattice planes of amorphous carbon, which is beneficial for $\mathrm{Li}^{+}$intercalation and deintercalation. ${ }^{17,45}$ The interlayer spacings of the three samples are summarized in Table 2. Moreover, COSDHC900-1 has sharper peaks than the other samples, suggesting increased crystallinity induced by the high temperature and carbonization period. This is also confirmed by the Raman spectra. Two prominent peaks located at $\sim 1360 \mathrm{~cm}^{-1}$ and $1590 \mathrm{~cm}^{-1}$ can be seen in Fig. 5b, corresponding to the $\mathrm{D}$ and $\mathrm{G}$ bands of stacked graphene, respectively. The $\mathrm{D}$ band represents the vibrational modes of disordered $\mathrm{sp}^{3}$ carbon caused by edges, defects, and disordered carbon sites, while the $\mathrm{G}$ band is related to the $\mathrm{E}_{2 \mathrm{~g}}$ vibration of $\mathrm{sp}^{2} \mathrm{C}$ atoms in graphene. The intensity ratio of the $\mathrm{D}$ band and $\mathrm{G}$ band $\left(I_{\mathrm{D}} / I_{\mathrm{G}}\right)$ can be used to reflect the degree of graphitization in carbon materials. The $I_{\mathrm{D}} / I_{\mathrm{G}}$ ratio of COSDHC900-1 is 0.92, significantly higher than that of COSDHC800-1 $\left(I_{\mathrm{D}} / I_{\mathrm{G}}=0.78\right)$ and COSDHC800-2 $\left(I_{\mathrm{D}} / I_{\mathrm{G}}=0.81\right)$, suggesting that more defects are present in the former sample, contributing to the reversible capacity of the anode and improving the Li storage ability. ${ }^{51}$

The cyclic voltammogram (CV) profiles of COSDHC900-1 recorded in the first three cycles with a scanning rate of $0.1 \mathrm{mV} \mathrm{s}^{-1}$ are shown in Fig. 6a. In the first cycle, the irreversible peak appears at $\sim 0.5 \mathrm{~V}$, corresponding to the formation of the solid electrolyte interface (SEI) layer on the surface of the electrode, ${ }^{52}$ which is assigned to the reaction between Li ions and 
Table 2 Interlayer distance, specific surface area and pore structure of COSDHC-T-H

\begin{tabular}{llllll}
\hline Sample & $2 \theta_{\mathrm{CO02}}\left({ }^{\circ}\right)$ & $d_{\text {002 }}(\mathrm{nm})$ & $S_{\mathrm{BET}}\left(\mathrm{m}^{2} \mathrm{~g}^{-1}\right)$ & $V_{\mathrm{t}}\left(\mathrm{cm}^{3} \mathrm{~g}^{-1}\right)$ & Fraction, \% (mesopore) \\
\hline COSDHC800-1 & 20.1 & 0.4414 & 545 & 0.321 & 85.7 \\
COSDHC800-2 & 20.96 & 0.4235 & 152 & 0.113 & 71.7 \\
COSDHC900-1 & 20.14 & 0.4405 & 1479 & 0.832 & 87.3
\end{tabular}
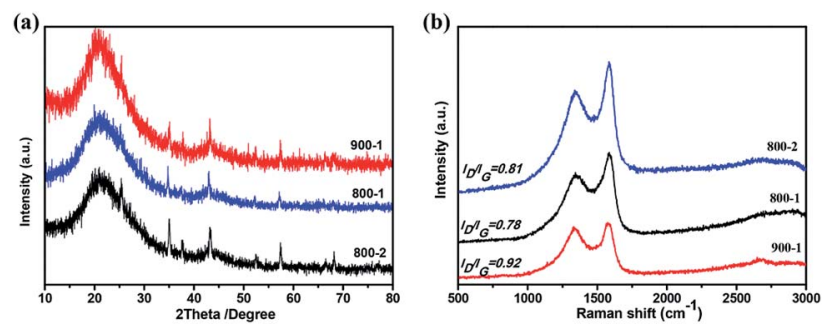

Fig. 5 (a) XRD patterns and (b) Raman spectra of COSDHC at different temperatures and carbonization periods.

the functional groups on the surface of COSDHC900-1. In subsequent cycles, the CV curves almost keep overlap, which indicates good reversibility with excellent capacity retention and electrochemical stability when $\mathrm{Li}$ is intercalated/delaminated. ${ }^{17}$ Fig. $6 \mathrm{~b}-\mathrm{d}$ show the 1 st, 2 nd, 50th, and 100th charge/ discharge profiles of COSDHC900-1, COSDHC800-1 and COSDHC800-2 electrodes, respectively, for LIBs at a current density of $200 \mathrm{~mA} \mathrm{~g}^{-1}$ with a voltage range of $3.0-0.01 \mathrm{~V}$. The
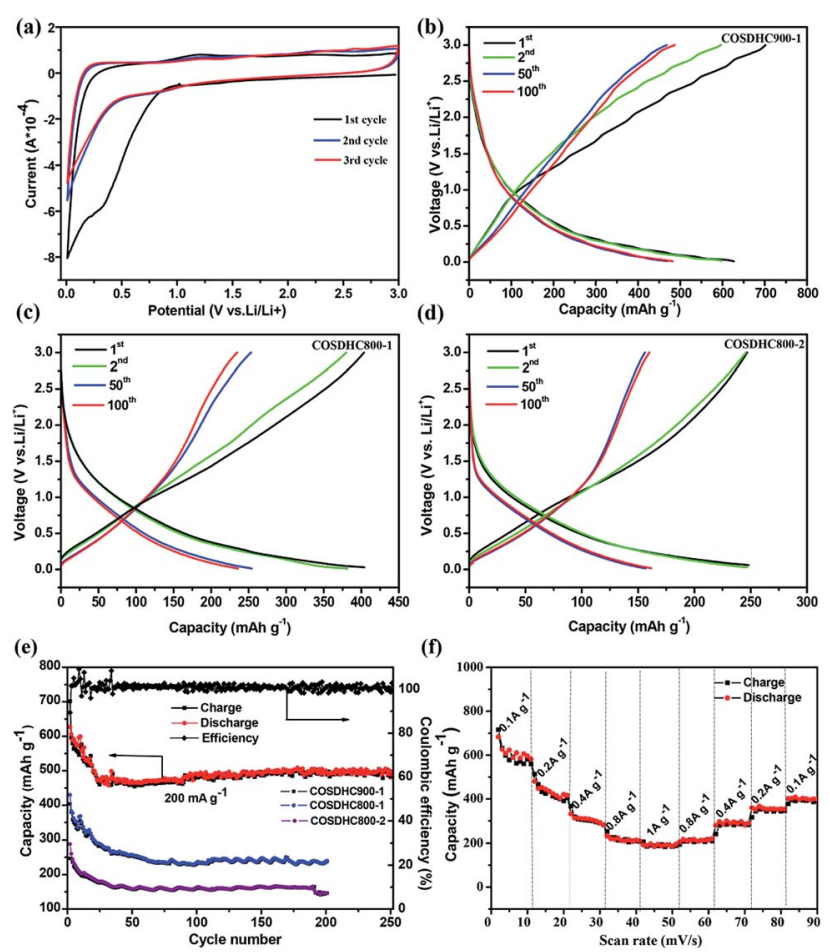

(f)

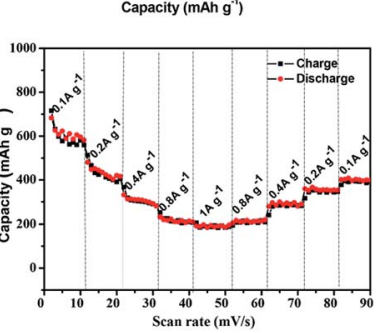

Fig. 6 (a) CV curves of COSDHC900-1; (b-d) discharge-charge profiles of COSDHC-T-H electrodes; (e) cycling performance of COSDHC-T-H electrodes with a current density of $200 \mathrm{~mA} \mathrm{~g}^{-1}$ and cycling between 0.01 and $3.0 \mathrm{~V} \mathrm{vs.} \mathrm{Li}^{+} / \mathrm{Li}$; (f) rate performance of COSDHC900-1. initial discharge capacities are 629.9, 429.9 and $294 \mathrm{~mA} \mathrm{~h} \mathrm{~g}^{-1}$, respectively. A remarkable difference exists in the specific capacity of carbon materials prepared at different temperatures and durations. After 50 cycles, the COSDHC900-1 sample retains a capacity of $473 \mathrm{~mA} \mathrm{~h} \mathrm{~g}{ }^{-1}$, but the other electrodes exhibit lower capacities of approximately $250 \mathrm{~mA} \mathrm{~h} \mathrm{~g}{ }^{-1}$ and $160 \mathrm{~mA} \mathrm{~h} \mathrm{~g}{ }^{-1}$. The specific capacity of COSDHC900-1 shows a gradual increase with increased cycles, indicating that the initially unexposed meso- and micropores become more accessible after long-term repeated cycling. ${ }^{53}$ Surprisingly, as shown in Fig. 6e, the COSDHC900-1 electrode delivers ultralong cycle stability when cycled at a current density of $0.2 \mathrm{~A} \mathrm{~g}^{-1}$ and has a higher capacity of $503 \mathrm{~mA} \mathrm{~h} \mathrm{~g}{ }^{-1}$, even after 250 cycles, with a coulombic efficiency of almost $100 \%$. Conversely, the other electrodes have lower capacities, mainly because of the low surface area and small number of active sites for the adsorption of Li ions due to low microporosity and mesoporosity. Fig. $6 \mathrm{f}$ shows the rate capacity of the COSDHC900-1 sample with 10 cycles at each rate (ranging from 0.1 to $1 \mathrm{~A} \mathrm{~g}^{-1}$ ), and the rate capacities of the COSDHC800-1 and COSDHC800-2 samples are shown in Fig. S4. $\dagger$ The reversible capacity of the COSDHC900-1 sample is $581,415,281,211$, and $195 \mathrm{~mA} \mathrm{~h} \mathrm{~g}^{-1}$ at $0.1,0.2,0.4$, 0.8 , and $1 \mathrm{~A} \mathrm{~g}^{-1}$, respectively. After the initial 10 cycles, the cell maintains good capacity retention during cycling at different current rates. However, after a high-current-density cycle, the COSDHC900-1 sample returns to a small current of $0.1 \mathrm{~A} \mathrm{~g}^{-1}$, and the capacity decreases to some extent $\left(424 \mathrm{~mA} \mathrm{~h} \mathrm{~g}^{-1}\right)$, which may be caused by the typical characteristics of biomass-derived hard carbon structures.

To further investigate the capacitive effect of the as-prepared COSDHC900-1 sample, we examined the electrochemical kinetics of the sample electrode for lithium storage by a series of CV measurements. Fig. 7a shows the stepwise CV curves of the COSDHC900-1 electrode under scan rates ranging from 0.1 to $10 \mathrm{mV} \mathrm{s}^{-1}$. The $\mathrm{CV}$ curves retain analogous shapes, and the peaks reflect pseudocapacitive behavior. In general, in CV curves, the peak current $(i)$ against scan rate $(\nu)$ is dominated by the equation $i=a \nu^{b}$, and the $b$ value can vary from 0.5 to 1 depending on the solvated ion storage mechanism. A $b$ value of 0.5 implies a diffusion-controlled process, while a value of 1.0 indicates that capacitive behavior dominates the charge storage process. ${ }^{10,45,54}$ In Fig. $7 \mathrm{~b}$, the linear relationship between the logarithmic sweep rate and logarithmic peak current is plotted, and the calculated $b$ value for anodic peak is 0.93 , suggesting fast kinetics due to a capacitive effect. Furthermore, the quantitative capacitive contribution at the given scan rate can be obtained from another equation: $i=k_{1} \nu+k_{2} \nu^{1 / 2}$, where $k_{1}$ and $k_{2}$ represent surface pseudocapacitive-controlled and diffusion- 

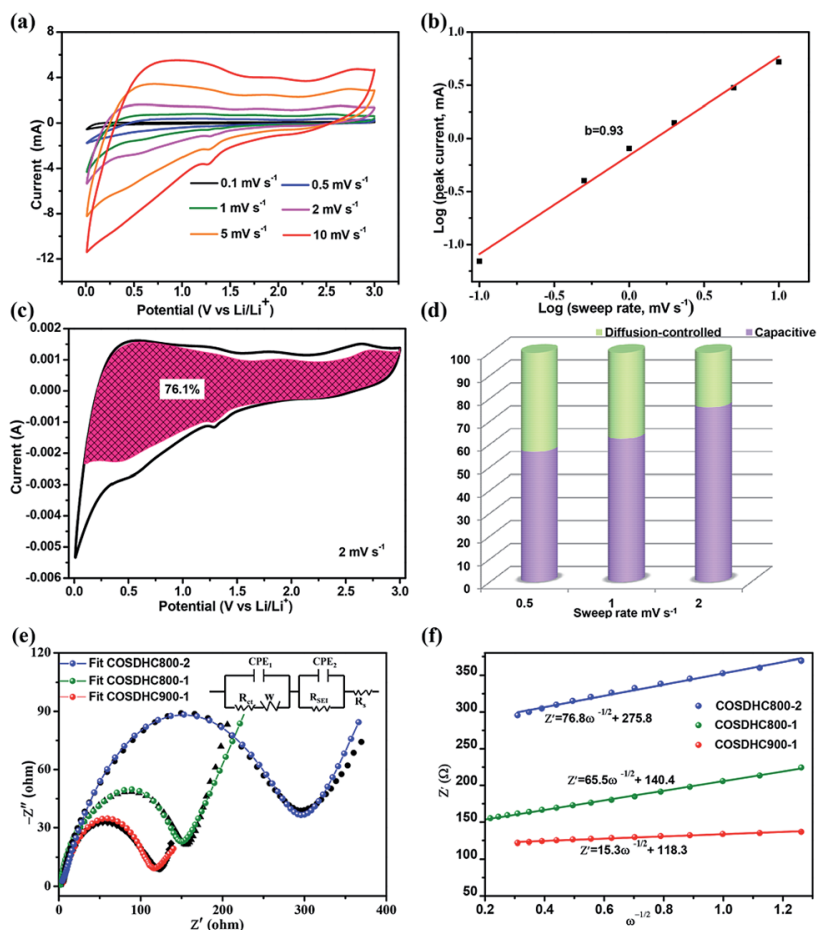

Fig. 7 (a) CV curves of COSDHC900-1 at different scan rates of 0.1, $0.5,1,2,5$ and $10 \mathrm{mV} \mathrm{s}^{-1}$, (b) $b$-values determined by the plot of log (i) vs. $\log (\nu),(\mathrm{c})$ capacitive contributions at a scan rate of $2 \mathrm{mV} \mathrm{s}^{-1}$, (d) capacitive contribution ratios at various rates, (e) Nyquist plots and equivalent circuits to simulate EIS results of COSDHC-T-H, (f) the relationship between $Z^{\prime}$ and $\omega^{-1 / 2}$ in the low-frequency region.

controlled processes, respectively. As a consequence, the capacitive contribution of the COSDHC900-1 anode is approximately $76.1 \%$ of the total capacity at $2 \mathrm{mV} \mathrm{s}^{-1}$ (as shown in Fig. 7 c). Fig. $7 d$ shows that the pseudocapacitive contribution percentage increases with increasing scan rate. The contributions are $56.7 \%, 62.4 \%$ and $76.1 \%$ at scan rates of $0.5 \mathrm{mV} \mathrm{s}^{-1}$, $1 \mathrm{mV} \mathrm{s}^{-1}$ and $2 \mathrm{mV} \mathrm{s}^{-1}$, respectively. Electrochemical impedance spectra (EIS) measurements were conducted to investigate the resistance at the electrode interface, as shown in Fig. 7e. The impedance spectra consist of two main zones: a depressed semicircle at high frequency and a sloping straight line in the low-frequency region. The kinetic parameters were obtained from the equivalent circuit (inset of Fig. 7e), where $R_{\mathrm{S}}$ represents the SEI film resistance, $R_{\mathrm{ct}}$ reflects the charge-transfer resistance at the interface, and $Z_{\mathrm{w}}$ is ascribed to the Warburg impedance. Moreover, the charge-transfer resistance data are listed in Table 3. The smallest semicircle is observed for the COSDHC900-1 electrode, which suggests that the charge transfer rate at the

Table 3 Charge transfer resistances and ion diffusion coefficients from the EIS test

\begin{tabular}{llr}
\hline Sample & $R_{\mathrm{ct}}(\Omega)$ & $D_{\mathrm{Li}^{+}}\left(\mathrm{cm}^{2} \mathrm{~s}^{-1}\right)$ \\
\hline COSDHC800-1 & 136 & $0.22 \times 10^{-13}$ \\
COSDHC800-2 & 280 & $0.3 \times 10^{-13}$ \\
COSDHC900-1 & 103 & $0.56 \times 10^{-12}$
\end{tabular}

electrode-electrolyte interface is fast and that the overall process achieves the best reaction kinetics. In the low-frequency region, the relationship between $Z^{\prime}$ and $\omega^{-0.5}$ (Fig. 7f) can be used to calculate the Li-ion diffusion coefficient ${ }^{55-57}$ according to the following equation:

$$
D_{\mathrm{Li}^{+}}=R^{2} T^{2} / 2 A^{2} n^{4} F^{4} C^{4} \sigma_{\mathrm{w}}{ }^{2}
$$

where $A, n, F, C, R$, and $T$ stand for the electrode area $\left(1.44 \mathrm{~cm}^{2}\right)$, reactive electron number per chemical formula, Faraday's constant (96500 $\left.\mathrm{C} \mathrm{mol}^{-1}\right)$, molar concentration of Li ions (1.0 $\left.\times 10^{-3} \mathrm{~mol} \mathrm{~cm}^{-3}\right)$, gas constant $\left(8.314 \mathrm{~J} \mathrm{~mol}^{-1} \mathrm{~K}^{-1}\right)$, and testing temperature (298 K), respectively. $\sigma_{\mathrm{w}}$ is the Warburg factor. The Warburg factor, $\sigma_{\mathrm{w}}$, can be derived from the following equation:

$$
Z^{\prime}=R_{\mathrm{s}}+R_{\mathrm{f}}+R_{\mathrm{ct}}+\sigma_{\mathrm{w}} \omega^{-0.5}
$$

where $\omega$ stands for the angular frequency. The fitted line slopes indicate $\sigma_{\mathrm{w}}$ values of $15.3,65.5$, and $76.8 \mathrm{~cm}^{2} \mathrm{~s}^{-1 / 2}$ for COSDHC900-1, COSDHC800-1 and COSDHC800-2, respectively. Thus, the Li-ion diffusion coefficients of COSDHC900-1, COSDHC800-1 and COSDHC800-2 were calculated to be $c a$. $0.56 \times 10^{-12}, 0.22 \times 10^{-13}$, and $0.3 \times 10^{-13} \mathrm{~cm}^{2} \mathrm{~s}^{-1}$, respectively. The lower charge transfer resistance and higher $D_{\mathrm{Li}^{+}}$of COSDHC900-1 guarantee excellent performance. It is clear that the structure of COSDHC900-1 enables a short ion-transport pathway with less resistance, leading to a higher rate capacity than that of the other two samples. These results are also consistent with the findings of long-term cycling performance.

\section{Conclusion}

In summary, a porous carbon material has been successfully synthesized from Camellia oleifera shells via $\mathrm{K}_{2} \mathrm{CO}_{3}$ impregnation and pyrolysis. After investigating the carbonization temperature and duration, it was found that the carbon sample pyrolyzed at $900{ }^{\circ} \mathrm{C}$ for $1 \mathrm{~h}$ demonstrates well-developed crosslinked mesoporous structures. This sample exhibits advanced electrochemical performance as an anode for LIBs and a continuous increase in specific capacity (as high as $503 \mathrm{~mA} \mathrm{~h} \mathrm{~g}^{-1}$, even after 250 cycles at a current density of $0.2 \mathrm{~A} \mathrm{~g}^{-1}$ ). To the best of our knowledge, this is the first report to use such biomass to prepare hard carbon materials as anode materials for Li-ion batteries. Moreover, the demonstrated comparatively facile synthesis process will make these electrodes both inexpensive and environmentally friendly. It is believed that these findings will benefit the electrochemical community from both economic and knowledge perspectives.

\section{Conflicts of interest}

There are no conflicts to declare.

\section{Acknowledgements}

This research was supported by the China Scholarship Council, Natural Science Foundation of Hunan (No. 2017JJ2040), the 
Australia Research Council and the University of Technology Sydney (UTS) through the Discovery Early Career Researcher Award (DECRA DE170101009), ARC Discovery Project (DP170100436), UTS Chancellor's Postdoctoral Research Fellowship project (PRO16-1893), and UTS Early Career Researcher Grants ECRGS PRO16-1304.

\section{Notes and references}

1 Y. Sun, Q. Wu and G. Shi, Energy Environ. Sci., 2011, 4, 11131132.

2 F. Zhang, T. Zhang, X. Yang, L. Zhang, K. Leng, Y. Huang and Y. Chen, Energy Environ. Sci., 2013, 6, 1623-1632.

3 F. Bella, A. B. Muñoz-García, F. Colò, G. Meligrana, A. Lamberti, M. Destro, M. Pavone and C. Gerbaldi, ACS Omega, 2018, 3, 8440-8450.

4 Y. Tang, Y. Zhang, W. Li, B. Ma and X. Chen, Chem. Soc. Rev., 2015, 44, 5926-5940.

5 M. Falco, L. Castro, J. R. Nair, F. Bella, F. Bardé, G. Meligrana and C. Gerbaldi, ACS Appl. Energy Mater., 2019, 2, 1600-1607.

6 L. Lefrançois Perreault, F. Colò, G. Meligrana, K. Kim, S. Fiorilli, F. Bella, J. R. Nair, C. Vitale-Brovarone, J. Florek, F. Kleitz and C. Gerbaldi, Adv. Energy Mater., 2018, 8, 1802438.

7 J. C. Arrebola, A. Caballero, L. Hernán, J. Morales, M. Olivares-Marín and V. Gómez-Serrano, J. Electrochem. Soc., 2010, 157, A791-A797.

8 W. Wei, Z. Wang, Z. Liu, Y. Liu, L. He, D. Chen, A. Umar, L. Guo and J. Li, J. Power Sources, 2013, 238, 376-387.

9 F. Colò, F. Bella, J. R. Nair and C. Gerbaldi, J. Power Sources, 2017, 365, 293-302.

10 X. Xu, R. Zhao, W. Ai, B. Chen, H. Du, L. Wu, H. Zhang, W. Huang and T. Yu, Adv. Mater., 2018, 30, 1800658.

11 W. Long, B. Fang, A. Ignaszak, Z. Wu, Y.-J. Wang and D. Wilkinson, Chem. Soc. Rev., 2017, 46, 7176-7190.

12 J. Wang, P. Nie, B. Ding, S. Dong, X. Hao, H. Dou, X. Zhang, J. Wang, P. Nie and B. Ding, J. Mater. Chem. A, 2016, 5, 24112428.

13 B. Hu, S.-H. Yu, K. Wang, L. Liu and X.-W. Xu, Dalton Trans., 2008, 40, 5414-5423.

14 B. Hu, K. Wang, L. Wu, S. H. Yu, M. Antonietti and M. M. Titirici, Adv. Mater., 2010, 22, 813-828.

15 A. Caballero, L. Hernan and J. Morales, ChemSusChem, 2011, 4, 658-663.

16 N. Moreno, Á. Caballero, J. Morales and E. RodríguezCastellón, J. Power Sources, 2016, 313, 21-29.

17 G. Zeng, B. Zhou, L. Yi, H. Li, X. Hu and Y. Li, Sustainable Energy Fuels, 2018, 2, 855-861.

18 L. Wang, Z. Schnepp and M. M. Titirici, J. Mater. Chem. A, 2013, 1, 5269-5273.

19 R. R. Gaddam, D. Yang, R. Narayan, K. Raju, N. A. Kumar and X. S. Zhao, Nano Energy, 2016, 26, 346-352.

20 W. Lv, F. Wen, J. Xiang, J. Zhao, L. Li, L. Wang, Z. Liu and Y. Tian, Electrochim. Acta, 2015, 176, 533-541.

21 P. Zheng, T. Liu, J. Zhang, L. Zhang, Y. Liu, J. Huang and S. Guo, RSC Adv., 2015, 5, 40737-40741.
22 Y. Liu, Z. Shi, Y. Gao, W. An, Z. Cao and J. Liu, ACS Appl. Mater. Interfaces, 2016, 8, 28283-28290.

23 Q. Feng, H. Li, Z. Tan, Z. Huang, L. Jiang, H. Zhou, H. Pan, Q. Zhou, S. Ma and Y. Kuang, J. Mater. Chem. A, 2018, 6, 19479-19487.

24 Q. Jiang, Z. Zhang, S. Yin, Z. Guo, S. Wang and C. Feng, Appl. Surf. Sci., 2016, 379, 73-82.

25 M. Armandi, B. Bonelli, F. Geobaldo and E. Garrone, Microporous Mesoporous Mater., 2010, 132, 414-420.

26 J. L. Goldfarb, G. Dou, M. Salari and M. W. Grinstaff, ACS Sustainable Chem. Eng., 2017, 5, 3046-3054.

27 Y. Li, C. Li, H. Qi, K. Yu and C. Liang, Chem. Phys., 2018, 506, 10-16.

28 K. Wang, N. Zhao, S. Lei, R. Yan, X. Tian, J. Wang, Y. Song, D. Xu, Q. Guo and L. Liu, Electrochim. Acta, 2015, 166, 1-11.

29 R. Satish, A. Vanchiappan, C. L. Wong, K. W. Ng and M. Srinivasan, Electrochim. Acta, 2015, 182, 474-481.

30 K. Yu, J. Li, H. Qi and C. Liang, Diamond Relat. Mater., 2018, 86, 139-145.

31 H. Wang, Z. Liu, Q. Liang, H. Zhong, G.-C. Han, S. Zhang and Z. Chen, J. Cleaner Prod., 2018, 197, 332-338.

32 Y. Ma, Waste Biomass Valorization, 2016, 8, 549-559.

33 A. A. Arie, H. Kristianto, I. Suharto, M. Halim and J. K. Lee, Adv. Mater. Res., 2014, 896, 95-99.

34 S. Jayaraman, A. Jain, M. Ulaganathan, E. Edison, M. P. Srinivasan, R. Balasubramanian, V. Aravindan and S. Madhavi, Chem. Eng. J., 2017, 316, 506-513.

35 J. i. Hayashi, T. Horikawa, I. Takeda, K. Muroyama and F. N. Ani, Carbon, 2002, 40, 2381-2386.

36 H. Yang, R. Yan, H. Chen, D. H. Lee and C. Zheng, Fuel, 2007, 86, 1781-1788.

37 I. I. Gurten, M. Ozmak, E. Yagmur and Z. Aktas, Biomass Bioenergy, 2012, 37, 73-81.

38 A. M. Puziy, O. I. Poddubnaya, A. Martínez-Alonso, F. SuárezGarcía and J. M. D. Tascón, Carbon, 2005, 43, 2857-2868.

39 H. D. Nguyen, T. T. Thuy Mai, N. B. Nguyen, T. D. Dang, M. L. Phung Le, T. T. Dang and V. M. Tran, Adv. Nat. Sci.: Nanosci. Nanotechnol., 2013, 4, 015016.

40 Y. Chen, B. Huang, M. Huang and B. Cai, J. Taiwan Inst. Chem. Eng., 2011, 42, 837-842.

41 Q. Shi, J. Zhang, C. Zhang, C. Li, B. Zhang, W. Hu, J. Xu and R. Zhao, J. Environ. Sci., 2010, 22, 91-97.

42 E. Köseoğlu and C. Akmil-Bașar, Adv. Powder Technol., 2015, 26, 811-818.

43 Y. Li, Z. Li and P. K. Shen, Adv. Mater., 2013, 25, 2474-2480. 44 D. Luo, P. Han, L. Shi, J. Huang, J. Yu, Y. Lin, J. Du, B. Yang, C. Li, C. Zhu and J. Xu, Appl. Surf. Sci., 2018, 462, 713-719. 45 Y. Mao, D. Hui, B. Xu, Z. Lin, Y. Hu, C. Zhao, Z. Wang, L. Chen and Y. Yang, Energy Environ. Sci., 2012, 5, 7950-7955.

46 L. Chen, Y. Zhang, C. Lin, W. Yang, Y. Meng, Y. Guo, M. Li and D. Xiao, J. Mater. Chem. A, 2014, 2, 9684-9690.

47 Z. Li, Z. Xu, X. Tan, H. Wang, C. M. B. Holt, T. Stephenson, B. C. Olsen and D. Mitlin, Energy Environ. Sci., 2013, 6, 871878.

48 M. Thommes, K. Kaneko, A. V. Neimark, J. P. Olivier, F. Rodriguez-Reinoso, J. Rouquerol and K. S. Sing, Pure Appl. Chem., 2015, 87, 1051-1069. 
49 D. W. McKee, Fuel, 1983, 62, 170-175.

50 R. K. Shilpa and A. Sharma, J. Appl. Electrochem., 2017, 48, 113.

51 N. Phattharasupakun, J. Wutthiprom, N. Ma, N. Chanlek and M. Sawangphruk, Electrochim. Acta, 2018, 286, 55-64.

52 K. Tang, L. Fu, R. J. White, L. Yu, M.-M. Titirici, M. Antonietti and J. Maier, Adv. Energy Mater., 2012, 2, 873-877.

53 B. Campbell, R. Ionescu, Z. Favors, C. S. Ozkan and M. Ozkan, Sci. Rep., 2015, 5, 14575.
54 Y. Guo, Y. Zhang, Y. Wang, D. Zhang, Y. Lu, R. Luo, Y. Wang, X. Liu, J.-K. Kim and Y. Luo, Electrochim. Acta, 2019, 296, 989-998.

55 X. Hu, Y. Li, G. Zeng, J. Jia, H. Zhan and Z. Wen, ACS Nano, 2018, 12, 1592-1602.

56 P. Hu, T. Zhu, X. Wang, X. Wei, M. Yan, J. Li, W. Luo, W. Yang, W. Zhang, L. Zhou, Z. Zhou and L. Mai, Nano Lett., 2018, 18, 1758-1763.

57 C. Wang, A. J. Appleby and F. E. Little, Electrochim. Acta, 2001, 46, 1793-1813. 